\title{
Evidence of Landau Damping in a Fluid Coupled with an Anharmonic Lattice
}

November 12, 2018

\author{
A. Ludu, E. Padilla, M. A. Q. Mazumder \\ Dept. Mathematics \& Wave Lab, Embry-Riddle Aeronautical University \\ Daytona Beach, FL 32114, USA
}

\begin{abstract}
The Landau damping effect was observed in collisionless plasma, as a microscopic resonant mechanism between electromagnetic radiation and the collective modes. In this paper we demonstrate the occurrence of the Landau damping at macroscopic scale in the interaction between water waves and anharmonic lattice of magnetic buoys. By coupling the Navier-Stokes equations for incompressible fluid with the nonlinear dynamics of an anharmonic magnetic lattice we obtain a resonant transfer of momentum and energy between the two systems. The velocity of the flow is obtained in the Stokes approximation with Basset type of drag force. The dynamics of the buoys is calculated in the surfactant approximation for a specific frequency, then we use Fourier analysis to obtain the general time variable interaction. After involving an integral Dirichlet transform we obtain the time dependent expression of the drag force, the interaction waves-lattice with a new term in the form of a Caputo fractional derivative. We compare the results of the model with experiments performed in a wave tank with free floating magnetic buoys under the action of small amplitude gravitational waves. This configuration can be applied in studies for the attenuation with resonant damping of rogue waves, storms or tsunamis.
\end{abstract}

\section{Introduction}

Although collisionless damping of plasma waves, predicted by Landau in 1946 [1] is now one of the bases of modern plasma physics, it was not until the early sixties that this effect was fully understood and accepted by the wider plasma physics community. The breakthrough came when the physical mechanism, namely, interaction of a plasma wave (for example Langumir waves [3]) with the resonant particles, had been elucidated in [2] and Landau damping had been observed 
experimentally [4]. Later, it was realized that such resonant damping is not a unique feature of plasma, but is just one manifestation of the universal phenomenon of wave damping or amplification in a medium with a continuous oscillation spectrum. For e review see for example [5].

Similar effects are now well known in such diverse fields as liquids with gas bubbles [6], superfluids [7], flashing of fire-flies [8], pacemaker cells controlling the beating of the heart [9], etc. Although the theory [10] as well as its remarkable physical interpretation [11] were developed at the same time as the resonant waveparticle interaction in plasma physics, a straightforward analogy between these effects was realized only recently in the context of the physical models for wind generated water waves $[10,12,14]$. According to this theory, a wave is generated by resonant energy transfer from the wind layer, which moves with a velocity equal to the phase velocity of the surface wave, a mechanism analogous to Landau damping. Various mathematical aspects of such resonant effects in fluids were reviewed [13]. The rigorous mathematical theory is based on solving the Cauchy problem for the evolution equation (VlasovPoisson equation) and proving estimates on the solution. The initial data issue was solved and Landau damping was mathematically established for the nonlinear Vlasov equation [15].

The paper is organized as follows. After Introduction, in Sec. 2 we present the theoretical model in three subsection. In subsection 2.1 we described the water waves generated in the wave tank. In subsection 2.2 we calculate the drag force from the water flow acting upon the buoys. In subsection 2.3 we study the nonlinear magnetic lattice of buoys, its resonant frequency and the anharmonic oscillations. In section 3 we evaluate the momentum transfer between the linear waves and the nonlinear chain of buoys and find the criteria for resonance and maximum transfer. In section 4 we describe the experimental settings, the experimental results and comparison with the calculations. The paper ends with a Conclusion section.

\section{The theoretical model}

In this section we demonstrate the possibility of resonant transfer of momentum in the interaction between the surface water waves and the nonlinear (anharmonic) lattice of magnetic buoys. Our approach, based on the analysis described in $[11,14]$, consists in calculating the force of the water waves on the buoys, followed by coupling this force with the nonlinear magnetic lattice dynamics. We consider a 2-dimensional $(x, z)$ open water channel of uniform depth $h$ (rigid bottom at $z=-h$ ) with free surface described by the function $z=\eta(x, t)$ and gravitational acceleration $\vec{g}=(0,-g)$. We have a one-dimensional chain of magnetic buoys freely floating at the water surface along $x$ axis. Each buoy in this chain has positive buoyancy, and consists in a small floating sphere attached to a cylinder of radius $R$ and height $H$. The cylinders have attached at the bottoms a permanent magnet with magnetization along the cylinder axis. In quiescent water $(\eta=0)$ all buoys take the vertical position, with the magnetic cylinder completely submerged, and the sphere floating tangent to water surface. Because all the buoys have equal dipole magnetic moments $\vec{m}$ oriented in the same way by construction (north pole towards floating ball) they collectively repel, and when at rest in mechanical equilibrium the buoys separate equidistantly along the chain. We consider a chain of $n$ magnetic buoys bounded left-right by stoppers $(x=0, x=n L$. Here $L$ is the equilibrium distance between two adjacent magnetic buoys. 


\subsection{Gravity water waves}

The water body is considered incompressible, inviscid and under gravitational force. We neglect the surface tension because the Bond number for our system is large enough

$$
B=\frac{g H^{2} \rho}{\sigma} \simeq 2 .
$$

The characteristic length is considered the height of the magnetic buoys $H \simeq 0.1 \mathrm{~m}$, and $\rho, \sigma$ are water density and surface tension coefficient, respectively. We also consider that the sizes of the magnetic buoys are much smaller than the size of the water tank, and the wavelength $\lambda$ of the surface waves. Linear gravity waves are generated at one end of the water tank with a system of pumps, and they propagate through the buoys chain.

When waves are generated in the water tank the magnetic buoys follow the water surface and they move in the vertical plane. We describe each buoy's position by the $\left(x_{i}(t), z_{i}(t)\right)$ coordinates, $i=1, \ldots, n$, with $x=0$ at the left end of the wave tank Each buoy can tilt in the $(x, z)$ plane with an angle $\theta_{i}$, see Fig. (1), measured from the local vertical. We assume that the buoys are floating together with the water surface, and we neglect possible vertical oscillations of buoys relative to the water surface. Nevertheless, the horizontal component of buoys' velocity can differ from the surrounding water velocity. In the linear wave approximation the surface of water has the shape

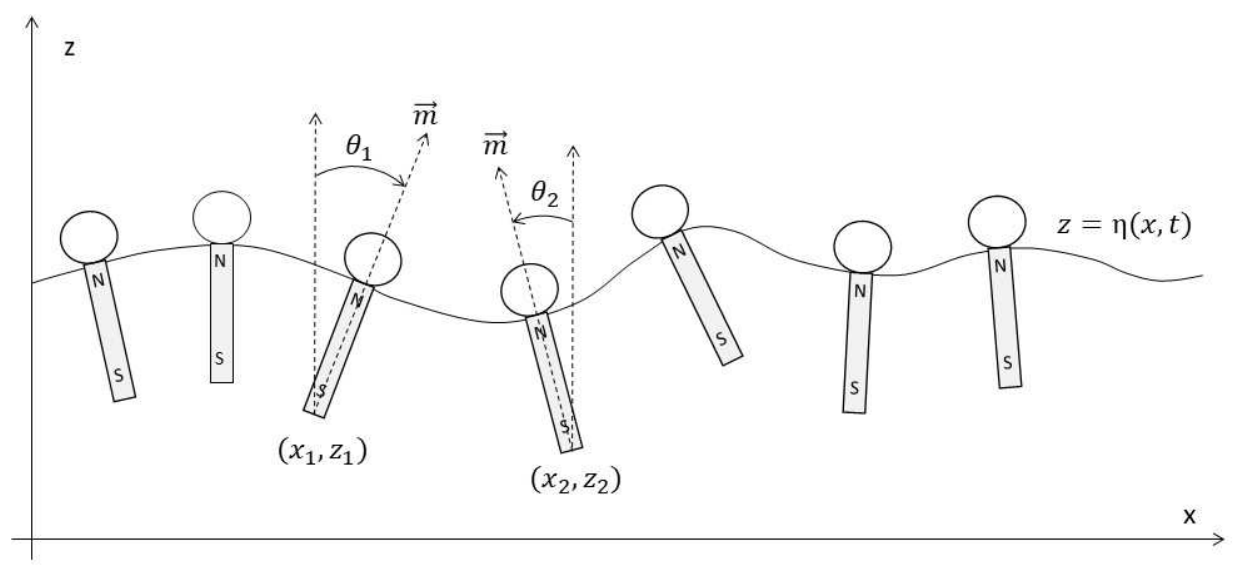

Figure 1: Magnetic buoys chain model at water surface. Each buoy is made of a floating sphere and a heavy magnetic cylinder. The magnetic dipoles $\vec{m}$ are identical and tend to repel each other when buoys are parallel and leveled. The motion of buoys is confined in the $(x, z)$ plane and each buoy can tilt an angle $\theta$. 
given by $[1]$

$$
\eta(x, t)=A \sin (\omega t-k x),
$$

with $A$ the wave amplitude, $k=2 \pi / \lambda$ the wave number, $\omega$ the wave angular velocity and with the dispersion relation $\omega=\sqrt{g k \tanh (h k)}$. The Eulerian velocity for such gravity waves $\vec{V}_{g}=\left(u_{g}, v_{g}\right)$ is given by

$$
\vec{V}_{g}=A \sqrt{\frac{2 g k}{\sinh (2 h k)}}(\sin (\omega t-k x) \cosh k(h+z), \cos (\omega t-k x) \sinh k(h+z)) .
$$

\subsection{Interaction between water waves and buoys}

The interaction between the traveling gravity waves generated in the wave tank and the floating chain of magnetic buoys results in the excitation of nonlinear modes of oscillations in this chain, which in their turn locally perturb the gravity waves flow around the buoys. By evaluating this perturbation effect on the initial waves we obtain the transfer of momentum from the waves to the chain of buoys, and from the rate of change of this momentum we find the drag force on the buoys. Let us consider a vertical buoy of radius $R$ and height $H$ placed vertically in a given flow field at a position $\vec{r}_{b}(t)$ measured from the absolute system of reference of the tank walls. The buoy has a absolute velocity $\vec{V}_{b}=\dot{\vec{r}}_{b}(t)$, and at the same point the fluid has an Eulerian velocity field $\vec{V}$. The absolute fluid velocity at a point far away from this buoy is the unperturbed gravity wave velocity field $\vec{V}_{g}$. In a relative frame of reference moving with $\vec{V}_{g}$ the velocity of the cylinder relative to the fluid is $\vec{U}=\vec{V}_{b}-\vec{V}_{g}$ which generates the drag.

The magnetic buoys, Fig. (2), have cylindrical shape of size $H=0.14 \mathrm{~m}, R=0.0127 \mathrm{~m}$ with rounded ends. This shape can be very well approximated with an ellipsoid of revolution with semi-axes $a=b=0.00635 \mathrm{~m}$ and $c=0.07 \mathrm{~m}$, such that the wave direction is along the $a$ semi-axis, and the $c$ semi-axis is vertical. For this geometry and size and under the action of small amplitude linear gravity waves the Reynolds number of the flow around the buoys is small $R e \simeq 500<4000$, so we can use the linearized Navier-Stokes equations for Stokes flow. Similar geometries involving

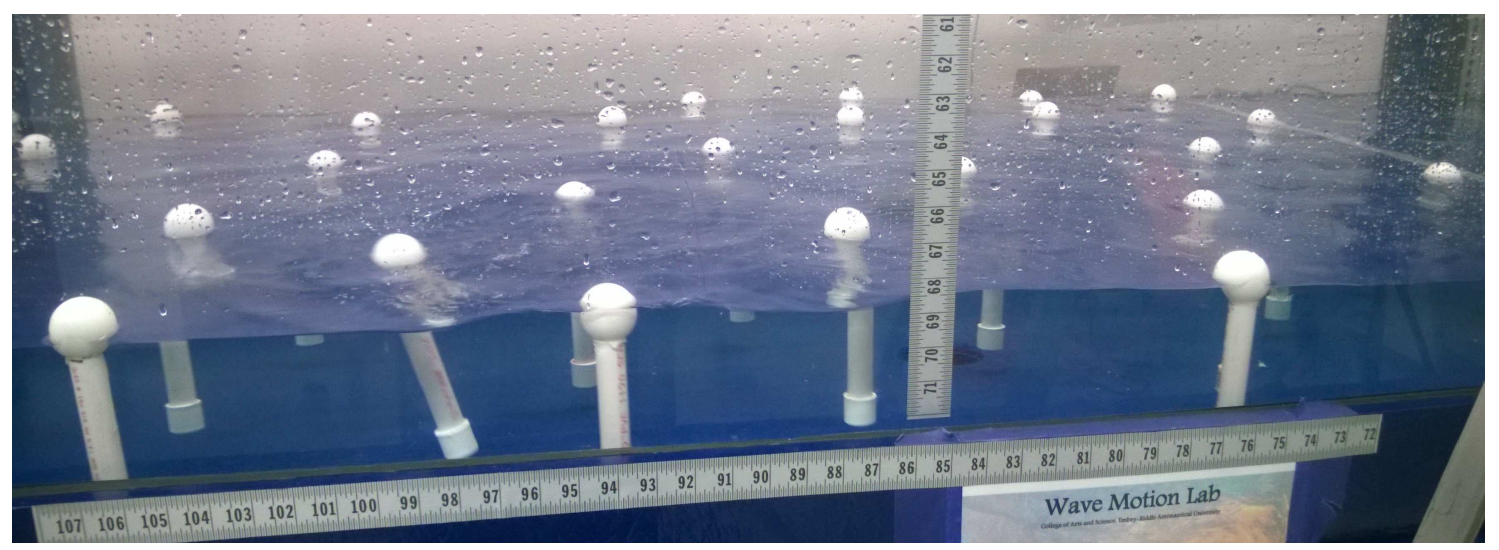

Figure 2: Experimental setting in the wave tank. A two-dimensional lattice with 100 magnetic buoys floating at the water surface of $h=0.3 \mathrm{~m}$ depth. The waves generated with a pump have $\lambda=1.45 \mathrm{~m}, \omega=50 \mathrm{~Hz}$, and amplitude $A=0.08 \mathrm{~m}$. 
finite cylinders were numerically tested using fully-coupled viscous fluid-solid interactions based on Navier-Stokes equations in either no slip boundary condition or the ghost-fluid technique and the nonlinear contribution to the flow was found negligible for similar range of velocities $[16,17]$. Similar conclusion were obtained in [18] where the authors used analytic calculations.

By taking all these in consideration, the relative flow around magnetic buoys is determined by the equation

$$
\mu \triangle \vec{V}-\nabla P+\vec{g}=0, \quad \nabla \cdot \vec{V}=0
$$

where $\mu$ is the dynamic viscosity and $P$ is the fluid pressure. By solving Eq. (3) we obtain the velocity distribution $\vec{V}$ around one isolated buoy. In the linear gravity waves we can consider that the fluid has a given constant and uniform horizontal velocity $\vec{V}_{\infty}=\left(V_{\infty}, 0\right)$ at infinity. By using this linear approximation together with the Stokes approximation, $[16,19]$, we obtain the velocity field in the form

$$
\vec{V}=\vec{V}_{\infty}+\nabla \times\left[\nabla \times\left(f \vec{V}_{\infty}\right)\right]
$$

where the function $f$ fulfills $\triangle^{2}(\nabla f)=0$, which actually reduces to the equation $\triangle^{2} f=0$ since all the flow field should be regular at infinity. This equation is solved with non slipping condition for the fluid at the buoy surface. To determine the pressure we substitute Eq. (4) in Eq. (3) and we have

$$
P=\mu \vec{V}_{\infty} \cdot \nabla(\triangle f)+P_{0}
$$

where $P_{0}$ is the local hydrostatic pressure. By using Eq. (5) for the pressure we can calculate the force $\vec{F}$ exerted on the buoy by the moving fluid, in the Stokes flow approximation, by integrating the fluid stress tensor $\sigma_{i k}$ over the surface $\Sigma$ of the buoy $[16,19]$

$$
F_{i}=\oint_{\Sigma}\left[-P \delta_{i k}+\left(\frac{\partial V_{i}}{\partial x_{k}}+\frac{\partial V_{k}}{\partial x_{i}}\right)\right] n_{k} d A
$$

In our model we consider the motion of the buoys in a so called "surfactant approximation", namely the buoys are vertically bound to the free fluid surface, and can move only by sliding along the surface. This hypothesis is natural for our system because the buoyancy force and the weight of buoys have a very stiff equilibrium at the free surface (with respect to the buoy sinking in the fluid), way steeper than the forces of drag or magnetic interactions. Hence the only degree of freedom for a buoy is the tangent motion along the fluid surface which, for small amplitude linear waves, can be approximated with the $x$-direction in the 2-dimensional flow case. Namely, the position of the buoy number $i$ in the chain is $\vec{r}_{b}=\left(x_{i}(t), z=\eta\left(x_{i}(t), t\right)\right)$. Under these approximations, by following [19], by using Eqs. (4,5) and integrating Eq. (6) along the ellipsoid surface we obtain the drag force along the $x$-direction, perpendicular on the buoys axis, in the form

$$
F_{x}^{\text {Stokes }}=\frac{16 \pi \mu a^{2} c}{\chi+a^{2} \alpha} V_{\infty}
$$

where we used the elliptic integrals

$$
\chi=a^{2} c \int_{0}^{\infty} \frac{d s}{\left(a^{2}+s\right)^{2} \sqrt{c^{2}+s}}
$$

and

$$
\alpha=a^{2} c \int_{0}^{\infty} \frac{d s}{\left(a^{2}+s\right) \sqrt{c^{2}+s}} .
$$


The result in Eq. (7) can be easily validate by using limiting situations. If $a=b=c=R$ we obtain from this equation the Stokes linear drag force for a sphere $6 \pi \mu R V_{\infty}$. Also in the case $a=0, b=c=R$ we obtain the drag for a disk moving broadside-on $16 \pi \mu R V_{\infty}$ and so on.

In the case when we consider fluid slipping at the buoy surface, with a coefficient $\beta$ of sliding friction, the boundary conditions change and we obtain the Basset drag force as a slipping correction to Eq. (7) [19]

$$
F_{x}^{\text {Stokes }}=\frac{16 \pi \mu a^{2} c}{\chi+a^{2} \alpha} \frac{\beta \alpha+2 \mu}{\beta \alpha+3 \mu} V_{\infty}
$$

Combining these expressions we obtain for the horizontal drag force on the cylindrical buoys the expression

$$
F_{x}^{\text {Stokes }}=\frac{32 \pi \mu\left(H^{2}-R^{2}\right)^{\frac{3}{2}}}{R^{2} \pi-2 H^{2} \pi+2 H \sqrt{H^{2}-R^{2}}+\left(4 H^{2}-6 R^{2}\right) \tan ^{-1} \frac{H}{\sqrt{H^{2}-R^{2}}}} V_{\infty}
$$

Further corrections to the Stokes's formula and a valid refinement of the flow pattern at short distances from the walls can be obtained by using Oseen's equation $[16,19]$

$$
F_{x}^{\text {Oseen }}=\frac{4 \pi \mu H}{\frac{1}{2}-\gamma-\ln \left(\frac{R \rho V_{\infty}}{4 \mu}\right)} V_{\infty}
$$

where $\gamma$ is Euler's constant.

The force expression changes if the fluid velocity is time variable. In the first step we consider the harmonic oscillation case of the flow, namely $\vec{V}_{\infty} \rightarrow \vec{V}_{\infty} e^{i \omega t}$. In this case [16] the biharmonic equation for the potential function $\triangle^{2} f=0$ involved in Eq.(4) has to be substituted with

$$
\triangle^{2} f+\frac{i \omega \rho}{\mu} \triangle f=0
$$

By solving Eq.(13) in the Stokes' approximation with no slipping boundary conditions on the buoys wall we obtain, after surface integration, the following oscillating horizontal drag force

$$
F_{x}^{o s c}=\frac{16 \pi \mu R^{2}}{\chi+R^{2} \alpha}\left(1+\frac{R}{\delta}\right) V_{\infty}+2 \pi R H \sqrt{\frac{2 \mu \rho}{\omega}}\left(1+\frac{R}{\delta}\right) \frac{d V_{\infty}}{d t}
$$

where $\delta=\sqrt{2 \mu / \omega \rho}$ is the depth of penetration of the transverse perturbation waves induced by the presence of the buoy in the free fluid motion. In the next step we consider an arbitrary time dependence of the asymptotic velocity $V_{\infty}(t)$. We expand this velocity in Fourier integral

$$
V_{\infty}(t)=\frac{1}{2 \pi} \int_{-\infty}^{\infty} V_{\omega} e^{-i \omega t} d \omega, \quad V_{\omega}=\int_{-\infty}^{\infty} V_{\infty}(\tau) e^{i \omega \tau} d \tau
$$

The total drag force will be the linear combination of the drag forces for each separate Fourier component, Eq. (14). By integrating with respect to $\omega$ back in the time domain, and by applying a Dirichlet transformation to change the order of integration we obtain the final form of the time dependent drag force

$$
F_{x}(t)=2 \pi \rho R^{3}\left[\frac{H}{R} \frac{d V_{\infty}}{d t}+\frac{8 \mu V_{\infty}}{\rho R\left(\lambda+R^{2} \alpha\right)}+\frac{H}{R^{2}} \sqrt{\frac{\mu}{\rho \pi}} \int_{-\infty}^{t} \frac{d V_{\infty}}{d \tau} \frac{d \tau}{\sqrt{t-\tau}}\right]
$$


The first term depends on the relative acceleration of the buoy in water and it represent the inertial transfer of energy from water to buoy, it is independent of the incompressible fluid properties and it is similar to the drag force in the case of potential flow, for inviscid fluids. The second term is the classical Stokes drag proportional to the velocity. The third term is the most interesting because it is actually the $1 / 2$ fractional derivative of the fluid velocity with respect to time. Indeed, the Caputo left-side fractional derivative [20]

$$
{ }_{t} D_{-\infty}^{\frac{1}{2}}\left[V_{\infty}(t)\right]=-\frac{1}{\Gamma(1 / 2)} \int_{-\infty}^{t}(t-s)^{-\frac{1}{2}} \frac{d V_{\infty}(s)}{d s} d s .
$$

The $1 / 2$ fractional derivative is exactly the half-way between first and second term, between zero and 1 derivative. Such type of operators are related to the memory of the process, so it is responsible for the history dependent process of transfer of momentum between fluid and the buoy. Usually, fractional calculus laws occur in fractal systems, or systems with variable memory, anomalous phase transition, etc., [21, 22], so it is very interesting how this exotic mathematical structure occurs naturally in a simple mechanical process.

\subsection{Anharmonic waves in the chain of magnetic buoys}

In this section we study the dynamics, waves and resonances in the one-dimensional chain of floating magnetic buoys. Networks of repelling magnets have been studied previously both theoretically and experimentally [24-26]. In all these studies, in general the magnets are suspended by long penduli and oscillate in gravitational field, or the magnets can slide frictionless along a bar. Given the nonlinear nature of the magnetic interaction the authors of these studies were mainly concern to study the existence of nonlinear waves, like solitary waves, breathers or solitons in these structures, and such highly localized stable waves were put into evidence and justified. In our case, we have the magnets freely float at the water surface, let them interact by the repelling force, and we couple this nonlinear lattice of free magnets with the surface waves generated from outside in a fluid tank. Our goal is to study the resonant transfer of energy and momentum from the water waves to the magnetic buoys chain. We demonstrate that under some conditions a strong resonant coupling and transfer of energy occurs like in the case of the microscopic Landau damping effect.

We have made experiments with both one-dimensional and two-dimensional lattices of magnetic buoys, but we performed the calculations only for the one-dimensional chain. In the following we elaborate only this system. Each buoy has a mass $M=80 \mathrm{~g}$ and enough buoyancy to float very

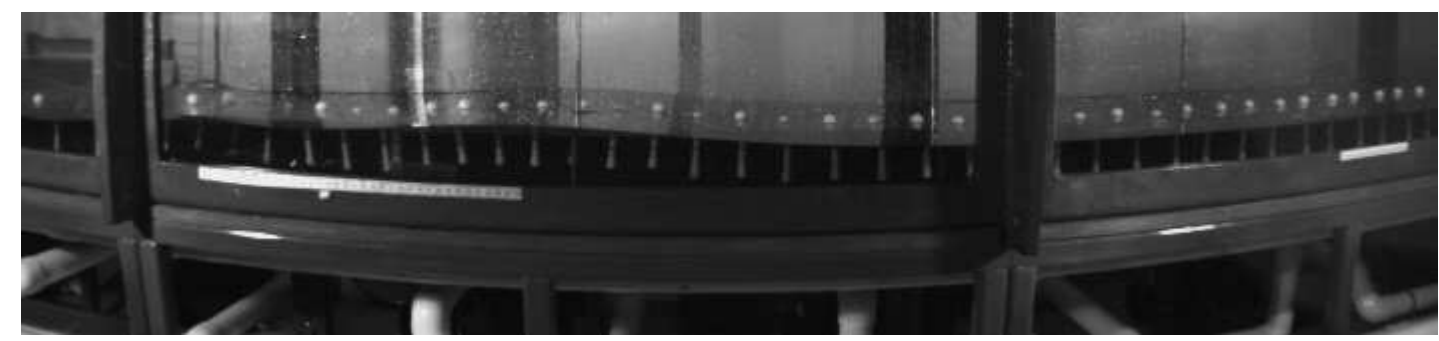

Figure 3: Gravity wave passing through the middle of the one-dimensional buoy chain. The water wave has amplitude $0.12 \mathrm{~cm}$ and wavelength $\lambda=1.6 \mathrm{~m}$. The nonlinear wave initiated in the magnetic chain has already arrived at the far right end. 
stable with the ping-pong ball at the upper end completely outside of water, but the whole cylinder holding the magnet at its base completely submerged. The magnets are grade N50 Neodymium cylindrical magnets ND062-0-N50, axially magnetized and nickel coated with height $24.5 \mathrm{~mm}$ and radius $12.25 \mathrm{~mm}$, axially magnetized at $B=13,200 \mathrm{Gs}$ with measured magnetic moment $m=$ $1.62 \mathrm{Nm} / \mathrm{T}$. Because the relative distances between the magnetic buoys are in general larger than the length of the magnetic dipoles, we consider only the magnetic dipole-dipole force and neglect the magnetic torque. However, the orientation of the buoys can be changed by the impact with water waves. In the following, we consider the buoys always moving along and together with the water surface, and having the magnetic dipoles normal to the water surface, see Fig. (1). The force upon a magnetic dipole $\vec{m}_{1}$ placed at origin generated by another magnetic dipole $\vec{m}_{2}$ placed in position $\vec{r}$ is given by [23]

$$
\vec{F}=\frac{3 \mu_{0}}{4 \pi r^{5}}\left[\left(\vec{m}_{2} \cdot \vec{r}\right) \vec{m}_{1}+\left(\vec{m}_{1} \cdot \vec{r}\right) \vec{m}_{2}+\left(\vec{m}_{1} \cdot \vec{m}_{2}\right) \vec{r}-\frac{5\left(\vec{m}_{1} \cdot \vec{r}\right)\left(\vec{m}_{2} \cdot \vec{r}\right) \vec{r}}{r^{2}}\right],
$$

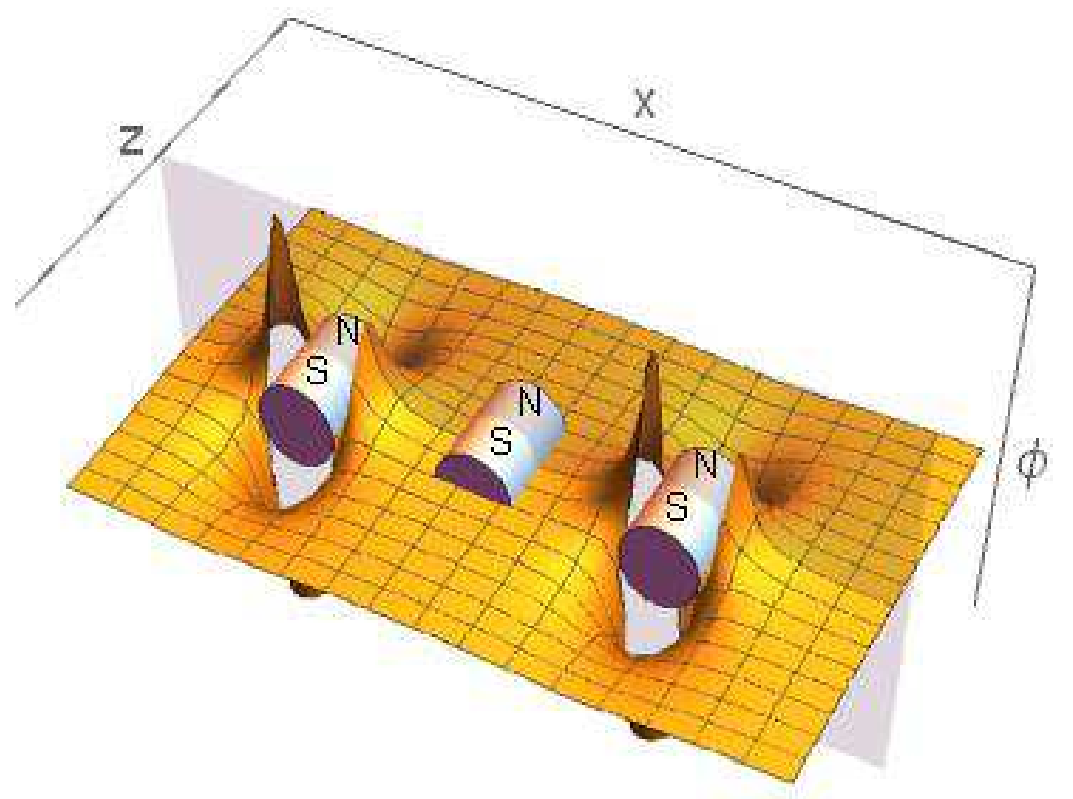

Figure 4: The meshed surface represents the magnetic potential $\Phi$ upon the central dipole, generated by the two peripheric magnetic dipoles. The surface of quiscent water is represented by the $z=0$ plane (veertical plane in the plot). Positive values for $\Phi(x, z)$ represent repelling between dipoles. 
where we use S.I. system of units and $\mu_{0}$ is vacuum permeability. The dipole force Eq. (17) is potential $\vec{F}=-\nabla \Phi$, and it results from the magnetic field generated by an infinitesimal magnetic dipole

$$
\vec{B}=\frac{\mu_{0}}{4 \pi}\left[\frac{3 \vec{r}(\vec{r} \cdot \vec{m})}{r^{5}}-\frac{\vec{m}}{r^{3}}\right]
$$

if the magnetic dipole is placed at origin and $r \neq 0$. Consequently, the magnetic potential of a dipole $\vec{m}_{2}$ placed in the origin under the field Eq. (18) of a dipole $\vec{m}_{1}$ is

$$
\Phi=-\vec{m}_{2} \cdot \vec{B}_{1}
$$

Let us consider two magnetic buoys with the same magnetic moment, mass and sizes. We place one magnetic buoy at the origin of the axes and we tilt it with angle $\theta_{1}$, while the other one is placed at a position $(x, z)$ and is tilt at an angle $\theta_{2}$. The potential of interaction between these two buoys has the form

$$
\Phi(x, z)=-\frac{m^{2} \mu_{0}\left[\left(x^{2}+z^{2}\right) \cos \left(\theta_{1}-\theta_{2}\right)-3\left(x^{2}-z^{2}\right) \cos \left(\theta_{1}+\theta_{2}\right)+6 x z \sin \left(\theta_{1}+\theta_{2}\right)\right]}{8 \pi\left(x^{2}+z^{2}\right)^{5 / 2}} .
$$

The chain we are using has stoppers at its ends, so that the end buoys cannot extend indefinitely. The $N$ buoys are also confined to move only along a direction, along the wave tank, by two very thin, tensioned and parallel carbon fibers with $2.5 \times 10^{6}$ PSI tensile elongation and 0.0023 static friction coefficient. When we line-up the buoys along this one-dimensional channel, and the water

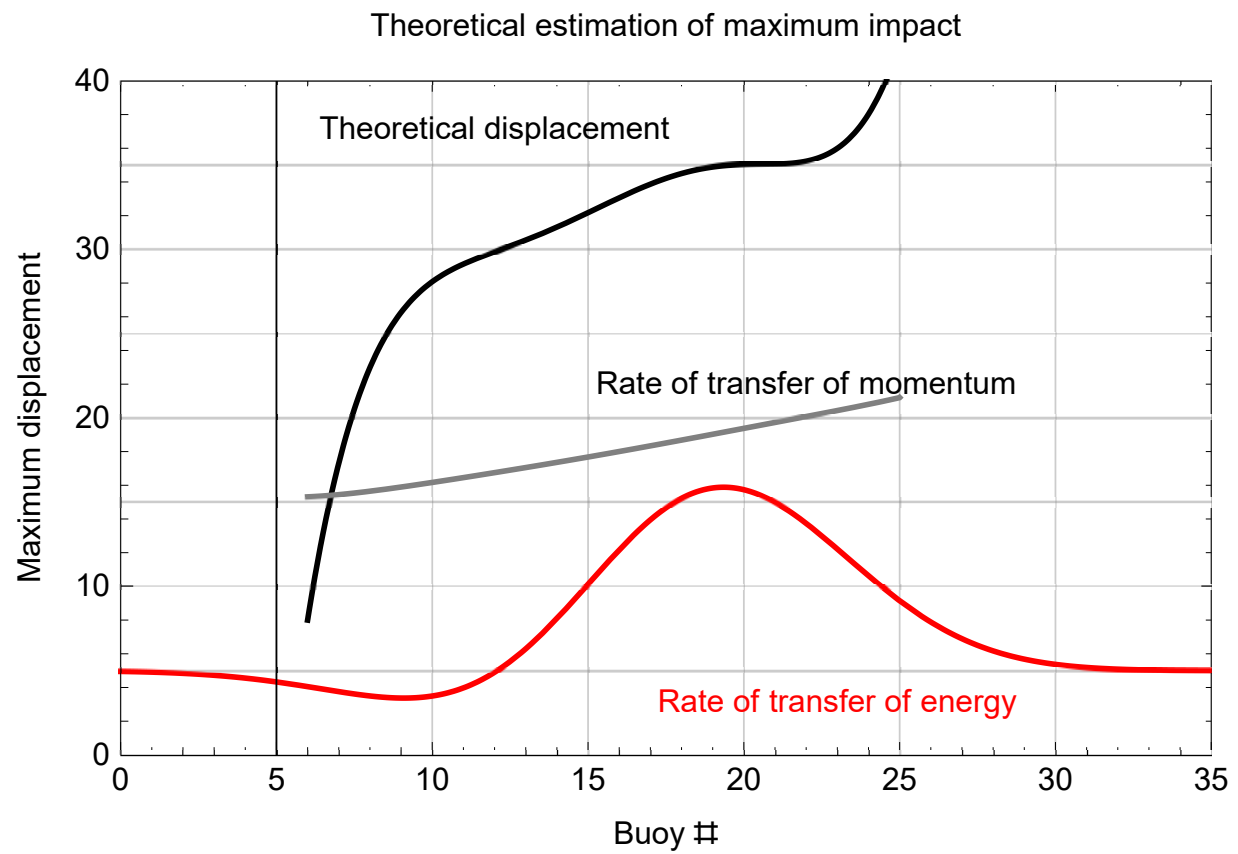

Figure 5: Black curve: Theoretical results for the displacement of the buoys under the action of the water wave. Gray curve: the rate of change of momentum between buoys and water. Red curve: The rate of change of energy between the buoys and water. 
is at rest, the buoys separate by magnetic repulsion at equal distances which we denote $L$. When the positions of the buoys are perturbed by an incoming water wave, a nonlinear wave is triggered in the chain and propagates, usually faster than the water perturbation. The general behavior of the nonlinear wave traveling in the nonlinear magnetic chain strongly suggested resonant coupling between adjacent particles in the chain leading to phase shifted motions. Small impulses spread at a constant speed, the resulting oscillations having maximum amplitude near the impulse site. Large impulses are more stable and evolved into stable wave packets concentrated over a fixed number of buoys and freely propagate along the chain Fig.(3).

We consider in this model that one buoy interacts only with its closest neighbors, and the magnetic force is horizontal. Let us assume that at moment $t$ the free surface of water has the linear wave shape $z=\eta(x, t)$. The horizontal component of the magnetic force acting on buoy number $i$ from its left and right neighbors is given by

$$
F_{i}(t)=-\frac{\partial}{\partial x_{i}}\left(\Phi\left(x_{i}-x_{i-1}, \eta\left(x_{i}, t\right)-\eta\left(x_{i-1}, t\right)\right)+\Phi\left(x_{i+1}-x_{i}, \eta\left(x_{i+1}, t\right)-\eta\left(x_{i}, t\right)\right)\right)_{x_{j}=x j(t)}
$$

for $j=i-1, i, i+1$. In Fig. (4) we present the potential energy from Eq. (21) of one buoy in the magnetic field of its two adjacent neighbors for parallel positions. Consider now the horizontal oscillations of the $i-$ th buoy in the chain considering that adjacent neighbors are fixed, that is

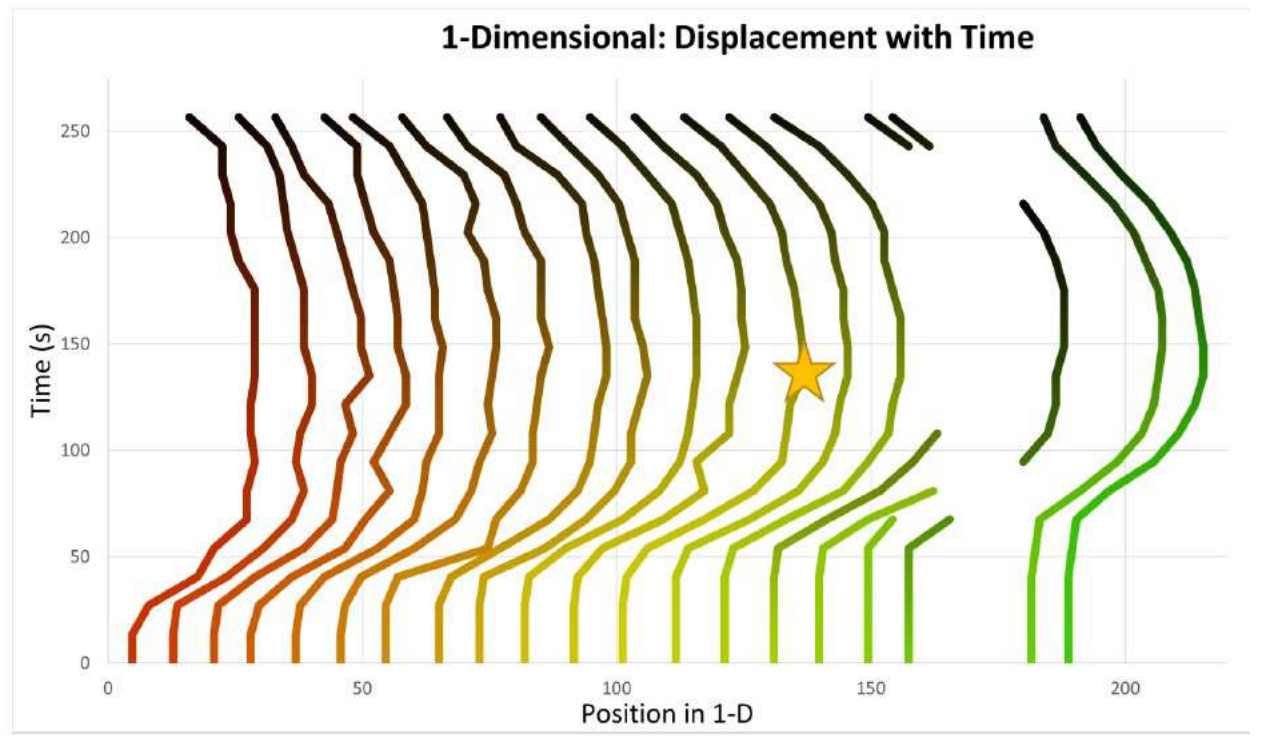

Figure 6: Space (horizontal axis) and time (vertical axis) evolution diagram of the one-dimensional chain of magnetic buoys. The water wave moves from left to right and put the buoys in motion. The paths 15-18 counted from left appear broken because it was no visibility to record their position. When the water wave displaced buoy 1 (from left), its the magnetic field triggered a nonlinear wave in the chain which accelerated in the same direction all the other buoys. By the time when the wave arrived at the last buoys to the right, these were already in motion. Conseqently they accepted the wave energy in a resonant process (the "surfer launching effect") and moved faster and at alrger amplitudes. 
$x_{i-1}=i L, x_{i+1}=(i+1) L$. Then the frequency of the corresponding buoy oscillations can easily be calculated and it reads

$$
\begin{gathered}
\omega_{b}^{2}=\frac{3 m^{2} \mu_{0}}{2 L^{6} \pi M}\left[L \cos \left(\theta_{1}-\theta_{2}\right)-3 L \cos \left(\theta_{1}+\theta_{2}\right)+L \cos \left(\theta_{2}-\theta_{3}\right)\right. \\
\left.-3 L \cos \left(\theta_{2}+\theta_{3}\right)+10\left(z_{2}-z_{1}\right) \cos \left(\theta_{1}+\theta_{2}\right)+10\left(z_{3}-z_{2}\right) \cos \left(\theta_{1}-\theta_{2}\right)\right],
\end{gathered}
$$

which reduces in the simplest case $\theta_{1}=\theta_{2}=\theta_{3}=z_{1}=z_{2}=z_{3}=0$

$$
\omega_{b}^{2}=\frac{3 m^{2} \mu_{0}}{\pi L^{5}} .
$$

This frequency of the fundamental linear mod of the chain is highly sensitive to the distance between buoys. For example, when the distance changes from $0.1 \mathrm{~m}$ to $0.15 \mathrm{~m}$ the frequency changes from $60 \mathrm{~Hz}$ to $2 \mathrm{~Hz}$. Consequently, when the water wave start to compress the chain its resonant frequency increases locally very quickly.

In order to obtain the group velocity of the nonlinear waves we have to expand Eq. (21) in Taylor series around zero with respect to $x_{i-1}, x_{i}, x_{i+1}$. If we denote by $C_{0}$ the Taylor coefficient

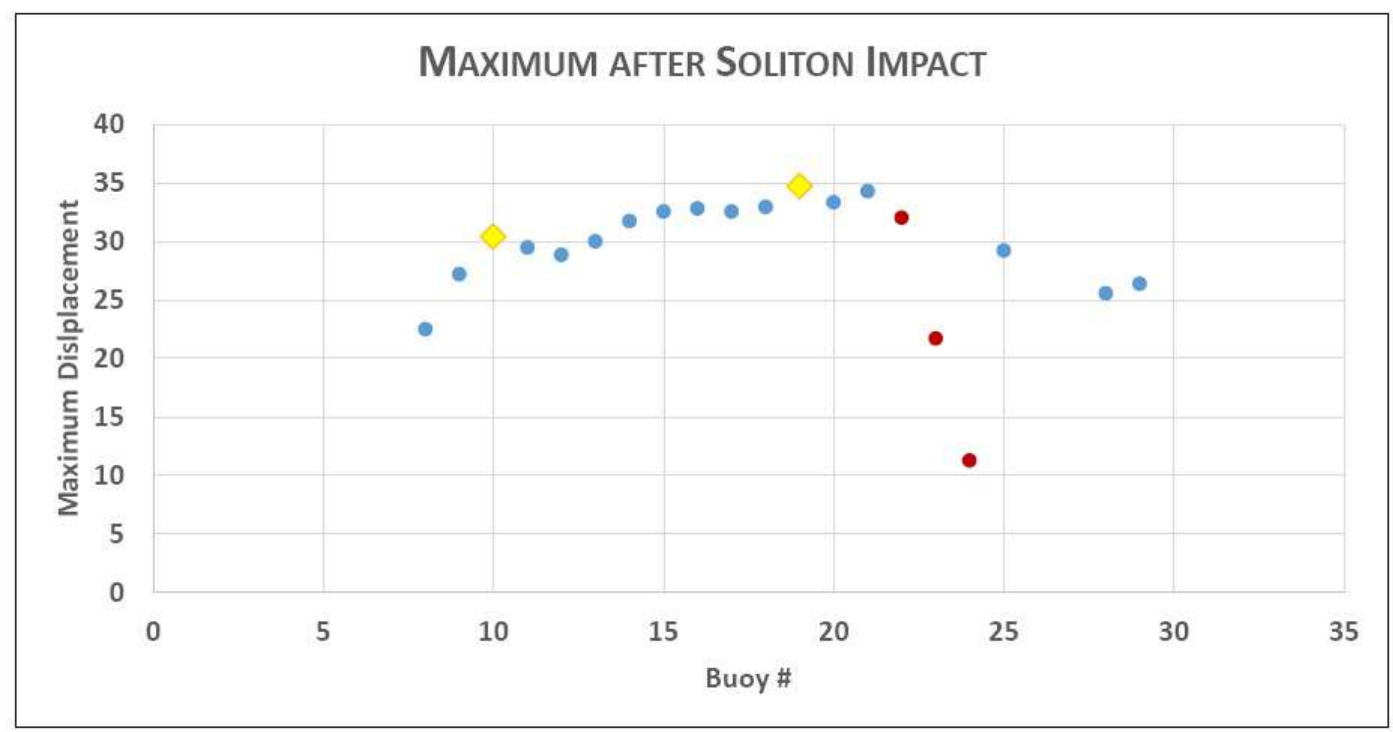

Figure 7: The blue dots represent the maximum horizontal displacement of various buoys in the chain. The yellow signs represent fixed markers in the wave tank the same as in Fig. (6). The brown dots represent buoys that accidentaly sticked magnetically together (north to south) because of too much vertical displacement. Each displacement is measured at the moment when the water wave arrived at that buoy. The number of the buoy shows its position along the $x$-axis. The further buoys were placed the more maximum wave horizontal displacement recorded because of the resonant energy transfer. These last buoys were already in motion because of the anaharmonic acceleration magnetic wave, so the water wave transfered momentum more efficiently to these ones. 
of $x_{i}$ and by $C_{1}$ the Taylor coefficient of the expression $x_{i+1}-x_{i-1}$ the dispersion relation is

$$
\omega_{b}^{2}\left(k_{b}\right)=C_{0}-2 C_{1}\left(1-\cos k_{b}\right)
$$

where $C_{0}=\omega_{b}^{2}$. From here we can extract the group velocity

$$
V_{\text {group }}=\left|\frac{d \omega_{b}}{d k_{b}}\right|=\frac{C_{1} \sin k_{b}}{\omega_{b}},
$$

and compare with experiments.

A more comprehensive dynamical analysis of this chain should take into account the shift in the buoys height because of the water wave, and the angles between the magnetic buoys because their tendency to line up perpendicular to water surface, and then perpendicular to the traveling water wave envelope. The strong dependence of the frequency, and hence the group velocity of waves through this chain makes very likely the enhancement of propagation of nonlinear compact waves like solitons or breathers [24-26]. An interaction between a soliton generated in the shallow water in the tank, and solitons exited in the nonlinear magnetic chain would be a very interesting subject for a future study.

\section{Resonant energy transfer}

In order to couple both systems we need to write the equation of motion of the buoys under the wave and magnetic forces. The rate of change of the momentum $M V_{i}$ of the buoy number $i$ is given by the drag force upon this buoy plus the magnetic interaction force with the first neighbors. The drag force is calculated from Eq. (15) where we substitute for $V_{\infty}$ the difference between the buoy Eulerian velocity and the velocity of flow under free gravitational waves given in Eq. (2). In the following equation we have only the $x$-component of the velocities involved

$$
M \frac{V_{i}}{d t}=2 \pi \rho R^{3}\left[\frac{H}{R} \frac{d\left(V_{i}-V_{g}\right)}{d t}+\frac{8 \mu\left(V_{i}-V_{g}\right)}{\rho R\left(\lambda+R^{2} \alpha\right)}+\frac{H}{R^{2}} \sqrt{\frac{\mu}{\rho \pi}} \Gamma(1 / 2)_{t} D_{-\infty}^{\frac{1}{2}}\left(\frac{d\left(V_{i}-V_{g}\right)}{d t}\right)\right]+F_{i}(t),
$$

with $F_{i}(t)$ from Eq. (21) and $V_{g}$ from Eq. (2) with the wave number equal to the one for the water waves $k=k_{w}$. By solving numerically this system we obtain the velocities of each buoy, and hence by integration both their path and maximum displacement, and the rate of transfer of momentum from the waves. The last quantity is nothing but the average over all buoys of the horizontal force.

In Fig. (5) we present the main model results. The theoretical maximal displacement of the buoys predicted by the equation of motion is in excellent agreement with the measured one from Fig. (7). The maximum displacement estimated for the buoys $10-20$ is in very good agreement with our predictions. The reason why the displacement of the end-of-chain placed buoys cannot be very well predicted is because of the influence, in these zone, or the proximity of the wave maker and the damping beach. The sum of all forces acting on the chain of buoys provides the rate of transfer of the momentum, and this is presented also in Fig. (5). We noticed that the last buoys in the chain have the best rate of transfer momentum, and this effect is a proof of the resonance between the accelerated motion under water waves and the magnetic interaction. Squaring the momentum distribution and also averaging over all the buoys provides the rate of transfer of the energy, plotted in red in Fig. (5). The resonant transfer for the already accelerated buoy is evidenced here. 


\section{Experiment and comparison with the model}

The magnetic buoys were made of water sealed PVC tubes having attached inside the bottom part a very strong cylindrical magnet, and the the upper end glued a ping-pong ball. This configuration make the buoy float very stable with the line of separation between the ping-pong ball and the tube exactly at the water free surface. We placed around 100 such buoys in the wave tank which has sizes $12 \mathrm{~m}$ long by $1.2 \mathrm{~m}$ wide and the water depth was between $0.1 \mathrm{~m}$ and $0.8 \mathrm{~m}$. At one end of the wave tank we have a system of $\mathrm{x}=$ six electric pumps that can generate a fast pulse of water pressure which generates in its turn a traveling wave along the tank. At the other end we have a dumping beach which attenuates the incoming waves, and prevents to some extent the reflections. This system could generate waves with amplitude between $0.02-0.25 \mathrm{~m}$, frequencies $0.8-9.4 \mathrm{~Hz}$, and wavelengths $\lambda=0.55-3.8 \mathrm{~m}$. The buoys were either placed in a long chain in between two elastic, parallel carbon fiber wires, or in a rectangular two-dimensional lattice. Four level capacitive analogue gauges were used to measure the water wave profiles with their signals recorded in an oscilloscope. The dynamics of the buoys was recorded by a high speed video camera with maximum 1,000 frame per second, and movie duration up to 10 seconds. The movie were played back frame by frame and the motion of each buoy measured.

We performed series of experiments with different waves and different setting for the buoys lattices. We excited linear gravity waves or solitons in the wave tank. In every experiment the dynamics of the buoys confirmed the existence of a resonant momentum transfer between the waves and the buoys. The main quantity of interest in all these experiments was the estimation of the resonant momentum and energy transfer between water waves and buoys. We measure and then we plotted the space-time evolution diagram of the one-dimensional chain of magnetic buoys, see Fig. (6). When the water wave arrives from the left the buoy number 1 is pushed to the right and this displacement triggers a nonlinear magnetic wave in the chain, and all buoys start the displacement. The slope of the locus of the inflection points of all observed paths in Fig. (6) represents the group velocity of the magnetic nonlinear wave. Comparison between this slope and the group velocity obtained from Eq. (25) shows a match within $10 \%$ error. We also measured the maximum displacement of each independent buoy, Fig. (7), and compared it with the calculations from our model, Fig (5), in an effort to identify the occurrence of resonances.

\section{Conclusions}

We developed a model for momentum and energy resonant transfer between an anharmonic lattice of magnetic buoys floating on the water surface, and linear waves in water. We build a model based on Stokes flow for small Reynolds numbers with Basset drag force upon the anharmonic lattice of buoys. We calculate the drag forces in the surfactant approximation for a specific frequency, then use Fourier analysis to apply it to a general time variable interaction. After involving an integral Dirichlet transform we obtain the time dependent expression of the drag force, the interaction waves-lattice. Interestingly, the dependence on acceleration of this force is introduced by a Caputo fractional derivative.

It is worth noting that the nature of the forces which provide this resonant water-wave plus magnetic-buoys interaction on the water surface is macroscopic. This aspect is different from the microscopic case of waveparticle interaction in collisionless plasma in the traditional landau damping. Nevertheless, in spite of this scale difference, the actual mechanisms responsible for the resonance are remarkably similar at different scales. Indeed, the net momentum transfer from an electromagnetic wave sent through a colisionless plasma to the free electrons originates from 
the bunching of resonant electrons which is correlated with the electric field: the density of these electrons is slightly higher where the field has one direction, and lower for the opposite case. In the macroscopic case of the excitation of the nonlinear magnetic lattice by surface waves, the momentum is transferred by the coupling between the stable modes of the gravity waves and the resonance with close frequencies from the oscillation spectrum of the anharmonic lattice. In both cases the correlations occur under the resonant condition, though for Landau damping in plasma the selection is in the velocity space of the electron distribution function, while for wave-buoy interaction it is in the coordinate space of the distribution of buoys on the surface.

\section{References}

[1] L. D. Landau, On the vibrations of the electronic plasma, Journal of Physics (USSR) 10 (1946) $25-34$.

[2] J. M. Dawson, On Landau damping, Physics of Fluids 4 (1961) 869874.

[3] I. Langmuir and L. Tonks, Oscillations in ionized gases, Physical Review 33 (1929) 195.

[4] J. H. Malmberg and C. B. Wharton, Collisionless damping of electrostatic plasma waves, Physical Review Letters 13 (1964) 184186.

[5] D. D. Ryutov, Landau damping: half a century with the great discovery, Plasma Physics and Control Fusion 41 (1999) A1-A12.

[6] D. D. Ryutov, Analog of Landau damping: the problem of sound-wave propagation in a liquid with gas bubbles, Soviet Physics JETP Letters 22 (1975) 215217.

[7] B. Fak, K. Guckelsberger, M. Korfer, R. Scherm, and A. J. Dianoux, Elementary excitations in superfluids, Physical Review B 41 (1990) 87328748.

[8] J. Buck, Synchronous rhythmic flashing of fireflies, Quarterly Review of Biology 63 (1988) 256289.

[9] A. T. Winfree, The Geometry of Biological Time, Springer-Verlag, New York, 1980.

[10] J. W. Miles, On the water surface wave generation by wind, Journal of Fluid Mechanics 3 (1957) 185198.

[11] M. J. Lighthill, Physical interpretation of the mathematical theory of wave generation by wind, Journal of Fluid Mechanics 14 (1964) 385398.

[12] S. G. Sajjadi and A. Ludu, Energy Transfer from Wind to Wave Groups: Theory and Experiment, Advances and Applications of Fluid Mechanics 20, 1 (2017) 21-74.

[13] A. V. Timofeev, Resonant effects in oscillations of non-uniform flows in continuous media, Reviews in Plasma Physics 17 (1993) 157238 (ed. B. B. Kadomtsev, Plenum, New York).

[14] G. E. Vekstein, Landau resonance mechanism for plasma and wind-generated water waves, American Journal of Physics 66, 10 (1998) 886-892.

[15] C. Mouhot, and C. Villani, On Landau damping, Acta Mathematica 207, 1 (2011) 29201. 
[16] L. D. Landau and E. M. Lifshitz, Fluid Mechanics, Elsevier, Burlington, 2009.

[17] B. Bouscasse, A. Colagrossi, S. Marrone and M. Antuono, Nonlinear water wave interaction with floating bodies in SPH, Journal of Fluids Structures 42 (2013) 112-129.

[18] D. G. Crowdy, Analytical solutions for uniform potential flow past multiple cylinders, European Journal of Mechanics B/Fluids 25 (2006) 459-470.

[19] H. Lamb, Hydrodynamics, Dover Publications, New York, 1932 (article 339 and 343a).

[20] E. C. de Oliveira and J. A. T. Machado, A Review of Definitions for Fractional Derivatives and Integral, Mathematical Problems in Engineering 238459 (2014) 1-6.

[21] V. E. Tarasov, Fractional Dynamics, Springer, Heidelberg, 201.

[22] A. Ludu and H. Khanal, Differential equations of dynamical order, Electronic Journal of Differential equations 24 (2017) 47-61.

[23] D. D. Jackson, Classical Electrodynamics, John Wiley \& Sons, New York, 1998.

[24] F. M. Russell, Y. Zolotaryuk, J. C. Eilbeck, Maving breathers in a chain of magnetic pendulums, Physical Review B 55 (1997) 6304-6308.

[25] M. Moleron, A. Leonard, C. Daraio, Solitary waves in a chain of repelling magnets, Journal of Applied Physics 115 (2014) 184901.

[26] A. Mehrem, N. Jimenez, L. Salmeron, X. Garcia-Andres, Ll. Garcia-Raffi, R. Pico, V. J. Sanchez-Morcillo, Nonlinear Waves in a Discrete Magnetic Lattice, Wave propagation in Periodic Structure, Phononics 0745 (2015) $3^{\text {rd }}$ International Conference on Phononic Crystals, Paris, France, May 31-June 5.

[27] E. Padilla, W. C. Wilson, and A. Ludu, Experimental Evidence of Landau Damping in a Fluid at a Macroscopic Scale, presentation at the Int. Conf. Recent Adv. Pure Appl. Math. (ICRAPAM 2017) May 11-15, 2017, Kusadasi-Aydin, Turkey. 